\title{
SGLT-2 inhibitors as promising therapeutics for non-alcoholic fatty liver disease: pathophysiology, clinical outcomes, and future directions
}

This article was published in the following Dove Press journal:

Diabetes, Metabolic Syndrome and Obesity: Targets and Therapy

\section{Naser Eddin Gharaibeh \\ Marie-Noel Rahhal \\ Leili Rahimi \\ Faramarz Ismail-Beigi}

Department of Medicine, Case Western Reserve University, University Hospitals Cleveland Medical Center, Cleveland, $\mathrm{OH}, 44106$, USA
Correspondence: Naser Eddin Gharaibeh Department of Medicine, Case Western Reserve University, University Hospitals Cleveland Medical Center, 10900 Euclid Ave., Cleveland, OH 44I06-495I, USA

Tel +l 4439838045

$\mathrm{Fax}+12168443120$

Email Nasergar@yahoo.com

\begin{abstract}
Nonalcoholic fatty liver disease (NAFLD) is increasingly recognized as a major expanding national and international health problem. Despite numerous investigations using a variety of therapeutic agents, the positive result on any single medication has not been established enough to gain widespread approval. This is in part related to concerns regarding side effects of agents, but is also related to the complex etiology of NAFLD. An often discussed question has been whether insulin resistance that is frequently present in those with NAFLD is a cause of NAFLD or is merely associated with the condition. Nevertheless, it is clear that a very high proportion of patients with NAFLD are obese, have elements of metabolic syndrome, or have type 2 diabetes (T2DM). Also, much progress has been made toward a better understanding of the pathophysiology of NAFLD. Life-style interventions resulting in weight loss remain the foundation for the prevention and treatment of NAFLD. In addition, agents such as Vitamin E and pioglitazone as well as other glycemia-lowering agents including Glucagon Like Peptide-1 (GLP-1) receptor agonists and Sodium Glucose Contransporter-2 inhibitors (SGLT-2i(s)) exhibit positive effects on the clinical course of NAFLD. This narrative review summarizes the current understanding of the diagnosis, epidemiology, and pathophysiology of NAFLD and specifically focuses on the efficacy of SGLT2i (s) as a potentially promising group of agents for the management of patients with NAFLD.
\end{abstract}

Keywords: fatty liver, insulin resistance, beta-oxidation, liver biopsy, de novo lipid synthesis

\section{Plain language summary}

Nonalcoholic fatty liver disease (NAFLD) and nonalcoholic steatohepatitis (NASH) are major growing national and international health problems. NAFLD is commonly associated with obesity, metabolic syndrome, type 2 diabetes. There is high insulin resistance that is irrespective of presence of diabetes. Hepatic lipid content is a function of food intake and fatty acid delivery from adipose tissue, de novo synthesis in liver (a process stimulated by elevated glucose and insulin levels), $\beta$ oxidation of fatty acids (a process stimulated by glucagon), and export of triglycerides from the liver by VLDL particles. Use of SGLT2 inhibitors has been shown to reduce insulin resistance, glucose and insulin concentrations while increasing glucagon levels and glucagon/insulin ratios, all changes that decrease liver fat content. Recent evidence suggests that use of SGLT2 inhibitors represents a promising new approach for the management of patients with NAFLD and NASH.

\section{Introduction}

Nonalcoholic fatty liver disease (NAFLD) refers to a wide spectrum of conditions with excessive deposition of lipids in the liver in the absence of alcohol abuse. The 
spectrum ranges from simple steatosis to steatohepatitis followed by advanced fibrosis and cirrhosis that can lead to hepatocellular carcinoma (HCC). ${ }^{1-3}$ Early, stable and minimal hepatic steatosis per se does not appear to have serious clinical implications. ${ }^{2}$ However, nonalcoholic steatohepatitis (NASH), defined as fatty liver plus hepatic damage with inflammation, elevated aminotransferase levels, and fibrosis, has been associated with higher incidence of cirrhosis and a risk for hepatocellular carcinoma. ${ }^{2}$ Additional important clinical implications of NAFLD are derived from the clinical burden of the associated metabolic comorbidities including obesity, hyperlipidemia, hypertension, metabolic syndrome, and type 2 diabetes (T2DM). ${ }^{3}$

Much research effort has been focused on finding one or more effective modalities for prevention and treatment of NAFLD; however, up to the present time, weight loss and exercise continue to be the cornerstone of interventions with proven benefit in patients with NAFLD. ${ }^{4,5}$ It has been shown that the more advanced the disease, the more weight loss is required to achieve a meaningful improvement. ${ }^{6}$ However, it is also well known that weight loss and exercise programs are difficult to maintain.

Based on better understanding of the pathophysiology of the disease, different therapeutic modalities, in addition to changes in lifestyle, have been explored for treatment of NAFLD; these target four different pathways: ${ }^{7}$ The first group targets hepatic fat accumulation resulting in reductions in metabolic stress and insulin resistance; pioglitazone is an example of this modality; ${ }^{8}$ the second group includes medications that are aimed at inhibiting oxidative stress and hepatic inflammation and injury; an example is the use of vitamin $\mathrm{E} ;{ }^{9}$ a third group targets the gastrointestinal system, including agents that promote weight loss (eg, orlistat), as well as fecal microbial transplantation; ${ }^{10,11}$ and finally, antifibrotic agents such as simtuzumab. ${ }^{7}$ In addition to the above, a newly described therapeutic strategy is to inhibit the Notch signaling pathway that appears to have a profibrotic role in development of NAFLD. ${ }^{12}$

To the best of our knowledge, none of the available studied therapeutics has been approved by the FDA $;{ }^{13}$ however, pioglitazone, Glucagon Like Peptide-1 (GLP-1) receptor agonists (GLP-1RA), and Vitamin E are considered reasonable options given their positive effect on NAFLD. ${ }^{14}$ Sodium glucose cotransporter-2 inhibitors (SGLT2i(s)) that, similar to GLP-1RA, were initially introduced as glycemialowering agents were found to have beneficial effects on cardiovascular, renal, and metabolic outcomes, including
NAFLD. ${ }^{15-18}$ And, use of GLP-1RA and SGLT2i(s) has resulted in a paradigm shift in the management of T2DM. ${ }^{19}$

This article is focused on the potential benefits from the use of SGLT2i(s) as a treatment modality for NAFLD. Benefits include weight loss with decreases in total and visceral fat, hepatic fat content, transaminase levels, blood glucose and insulin levels and insulin resistance; additional benefits include improvements in metabolic and inflammatory parameters, and possibly lower rates of liver fibrosis and HCC. ${ }^{15,16,20-23}$ To prepare this analysis, PubMed was searched for the published literature in English from 1990 to 2019 for the pathogenesis, diagnosis, and treatment of NAFLD, and for SGLT2i(s) and their use in patients with NAFLD. Based on this search, relevant references were selected for this analysis.

\section{Diagnosis of NAFLD and modes of evaluation}

NAFLD is commonly associated with components of metabolic syndrome including central obesity, insulin resistance, hypertension, and hypertriglyceridemia as well as T2DM. ${ }^{14}$ Generally, patients with NAFLD are asymptomatic and are diagnosed incidentally by presence of mild to moderate elevations in alanine transaminase (ALT) and aspartate transaminase (AST) levels (and ALT/AST ratio of greater than 1.0), as well as increased serum ferritin. ${ }^{24}$ Of note, elevated liver enzymes underestimate the diagnosis of NAFLD when compared to non-invasive imaging techniques, and the degree of elevation of liver enzymes do not correlate with the extent of inflammation or fibrosis. ${ }^{3,24}$ Liver biopsy is the gold standard test for the diagnosis of NAFLD and for staging the liver disease. ${ }^{14}$ NAFLD is defined as more than 5\% steatotic hepatocytes in a liver tissue section. To evaluate the extent of steatosis in the liver, a commonly used method assesses the percentage of hepatocytes that are steatotic using mild ( $0-33 \%$ of hepatocytes), moderate $(33-66 \%)$, or severe $(>66 \%)$ steatosis. ${ }^{25}$

Several non-invasive imaging methods have been developed to evaluate the degree of hepatic steatosis. Ultrasonography is a screening technique that typically shows a diffuse increase in echogenicity in fatty liver; ${ }^{26}$ it is not as specific or sensitive compared to other imaging techniques especially in morbidly obese patients. ${ }^{27}$ Transient elastography detects and quantifies steatosis and fibrosis by measuring ultrasound signals emitted from the FibroScan probe. ${ }^{26}$ Magnetic resonance imaging (MRI) and magnetic resonance spectroscopy (MRS) are 
considered the most accurate imaging modalities for estimation of intrahepatic fat content. ${ }^{15,16}$

Scoring systems have also been developed for estimation of the degree of liver fibrosis. Frequently used are the FIB-4 index (Fibrosis Index Based on 4 factors: using age, AST, ALT, and platelet levels), ${ }^{26}$ the NAFIC score (NASH Ferritin and type IV collagen $7 \mathrm{~S}$ : using levels of ferritin, fasting insulin, and T4C7S [Type 4 Collagen $7 \mathrm{~S}$ ]), ${ }^{28}$ or the NFS score (NAFLD Fibrosis Score: using age, BMI, AST/ ALT ratio, serum glucose, and albumin). ${ }^{24}$ The above information can also be used to select patients in whom liver biopsy is needed for further classification, management, and follow-up.

\section{Epidemiology}

NAFLD is a common condition with a global prevalence of approximately $25 \%$; the highest prevalence is in the Middle East and South America and the lowest is in Africa., 34,29 Approximately one-fourth of individuals with NAFLD progress to non-alcoholic steato-hepatitis (NASH) over a period of 3-7 years, ${ }^{29}$ and in some the disease progresses to hepatocellular carcinoma (HCC). Due to the pandemic of obesity and T2DM, NAFLD has become the most common cause of liver disease worldwide. ${ }^{29}$ The prevalence of T2DM has been reported as $22.5 \%$ among patients with NAFLD and $43.6 \%$ among patients with NASH. ${ }^{3,30}$ The continuous hepatic inflammation can result in cirrhosis and ultimately increases the risk of HCC. ${ }^{31}$ This may explain the reported two-fold increase in the risk of HCC in obese individuals. ${ }^{32}$

It has been proposed that development of advanced fatty liver disease/steatohepatitis requires a "double hit": the first resulting in excessive hepatic fat accumulation, the second, stressful oxidative injury that initiates lipid peroxidation and fibrosis. ${ }^{33}$ This concept provides an essential thesis for approaches aimed at prevention and treatment of NAFLD. It may also explain the discrepancy between the higher prevalence of the commonly observed early fatty liver versus the more advanced stages of NAFLD and NASH. ${ }^{33}$

\section{Pathophysiology of NAFLD}

It is well established that fatty liver is a common finding in obese individuals with metabolic syndrome and in patients with T2DM, and that individuals with fatty liver exhibit increased insulin resistance (IR) even in the absence of T2DM. $^{34}$ IR is considered to be a critical pathophysiologic mechanism underlying NAFLD; this understanding is based on results of studies in rodent models and humans with multiple proposed mechanisms including augmented inflammatory state, decreased ability to reverse cell injury, and hepatic fat accumulation. ${ }^{14,21,35-37}$ Kumashiro and others evaluated the effect of these potential etiologies in morbidly obese patients with insulin resistance in the absence of T2DM using liver biopsy specimens obtained during bariatric surgery. ${ }^{21}$ Inflammatory and endoplasmic reticulum stress markers in addition to hepatic fat content were evaluated. Only accumulation of diacyl glycerol (DAG) - and not total hepatic lipid content - was associated with an increase in systemic IR. ${ }^{21}$ Further, it was found that accumulation of the hepatic DAG results in activation of Protein Kinase $\mathrm{C}$ (epsilon) which in turn leads to increased hepatic IR. ${ }^{35}$ Traditionally, systemic IR has been thought to lead to NAFLD; however, recent evidence indicates that NAFLD could be the inciting etiology of systemic and hepatic IR, not the reverse. ${ }^{35,38}$ Nevertheless, it is likely that once the process is initiated, the two mechanisms augment and amplify each other. ${ }^{39}$

Many studies have focused on the mechanisms leading to fatty liver. Increased fat deposition in the liver can result from one or a combination of the following mechanisms: a) increased delivery of lipids to the liver from high dietary intake or from increased release of fatty acids from an expanded adipose tissue mass, b) increased de novo synthesis of triglycerides and cholesterol in the liver, c) decreased hepatic oxidation of fatty acids, and d) decreased export of triglycerides from the liver (Figure 1). ${ }^{40,41}$ We will discuss these steps briefly to help gain a better understanding of how treatment with SGLT2 inhibitors can counteract some of these steps to alleviate NAFLD.

As shown in Figure 1, in healthy individuals, dietary triglycerides in chylomicrons are removed by the liver and deposited. Likewise, there is a constant flow of non-esterified free fatty acids emanating from adipose tissues, a process that is inhibited by insulin; in liver, the fatty acids are esterified to triglycerides and deposited in lipid-containing vesicle. ${ }^{40} \mathrm{~A}$ second pathway involves de novo synthesis of lipids and is mediated by both glucose and insulin. Following entry of glucose into hepatocytes and its phosphorylation, certain derivatives of glucose-6-phosphate activate the transcription factor carbohydrate response element protein (ChREBP). ${ }^{42}$ Activation of ChREBP stimulates the synthesis of several enzymes of lipid synthesis. Separately, phosphorylation of insulin receptors by insulin leads to activation of sterol regulator element-binding protein-1c (SREBP-1c) that in turn stimulates the transcription of genes involved in lipid synthesis. ${ }^{40}$ The third mechanism for control of hepatic 


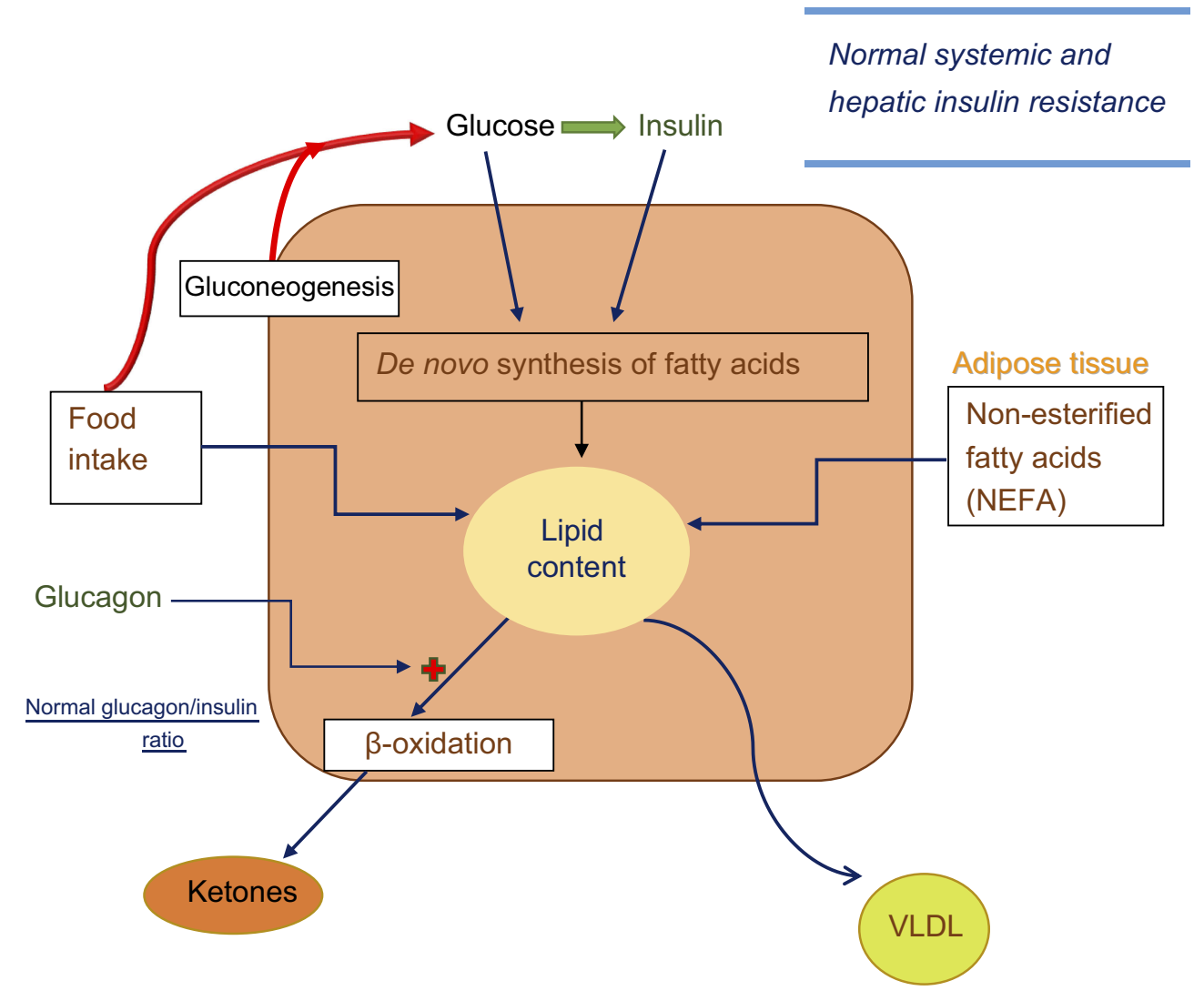

Figure I Lipid synthesis and deposition in hepatocytes in normal individuals. Blood glucose level is determined by food intake and endogenous glucose production from hepatic gluconeogenesis and glycogenolysis. Systemic and hepatic insulin resistance is normal. Increased glucose levels stimulate insulin secretion and increase blood insulin levels. Glucose and insulin each stimulate a cascade of metabolic events in liver to increase de novo fatty acid and cholesterol synthesis. In addition, hepatic lipid content is directly related to quantity and composition of food, and uptake of circulating non-esterified fatty acids released from adipose tissue. On the other hand, hepatic betaoxidation (and ketogenesis) that can be activated by glucagon, and export of very low-density lipoprotein (VLDL) are the main mechanisms through which liver fat content is decreased. Blood glucagon/insulin ratio is normal.

liver content is $\beta$-oxidation of fatty acids. This pathway is sensitive to stimulation by glucagon, ${ }^{37,43}$ and increased $\beta$ oxidation of fatty acids can lead to elevation of blood ketones. $^{36,37}$ Finally, export of hepatic triglycerides through VLDL transport supplies energy to peripheral tissues and helps decrease the level of hepatic lipids. ${ }^{40,41}$

Disturbances in the above pathways that can result in excessive fat deposition in the liver and lead to fatty liver and NAFLD are depicted in Figure 2. Here, multiple dysregulations are present. Increased food intake especially in obese individuals and increased fatty acids released from the expanded adipose tissue (and decreased insulin action due to high insulin resistance in adipose tissue) deposit extra lipids in hepatocytes. ${ }^{41}$ Chronically elevated insulin levels in response to high glucose levels in T2DM and prediabetes as well as to the increased systemic insulin resistance stimulate de novo lipid synthesis. ${ }^{40}$ There is slight (or no) increase in the rate of $\beta$-oxidation while glucagon levels are somewhat elevated and the glucagon/ insulin ratio is somewhat decreased. ${ }^{44}$ Finally, although there is an increase in VLDL synthesis and release by the liver, the magnitude of this change appears not to be large enough to overcome the increased rate of lipid deposition. $^{40,42}$ In the following sections, we will discuss the actions of SGLT2i(s) and their effects on counteracting some of the above underlying abnormalities (Figure 3 ).

\section{SGLT-2 co-transporter, its inhibitors, and their role in hepatic lipid metabolism}

Sodium Glucose cotransporter-2 (SGLT-2) is highly expressed in proximal tubular cells of the kidney and plays an essential role in reabsorption of filtered glucose. ${ }^{45}$ Expression of SGLT2 appears to be limited to the kidneys, with the exception of its expression in alpha cells of pancreatic islets. ${ }^{43,46}$ There is little or no expression in other tissues. ${ }^{45,47}$ SGLT2i(s) have been approved for use in patients with T2DM; they cause glycosuria and osmotic diuresis, reduce body and fat mass, reduce blood pressure, 


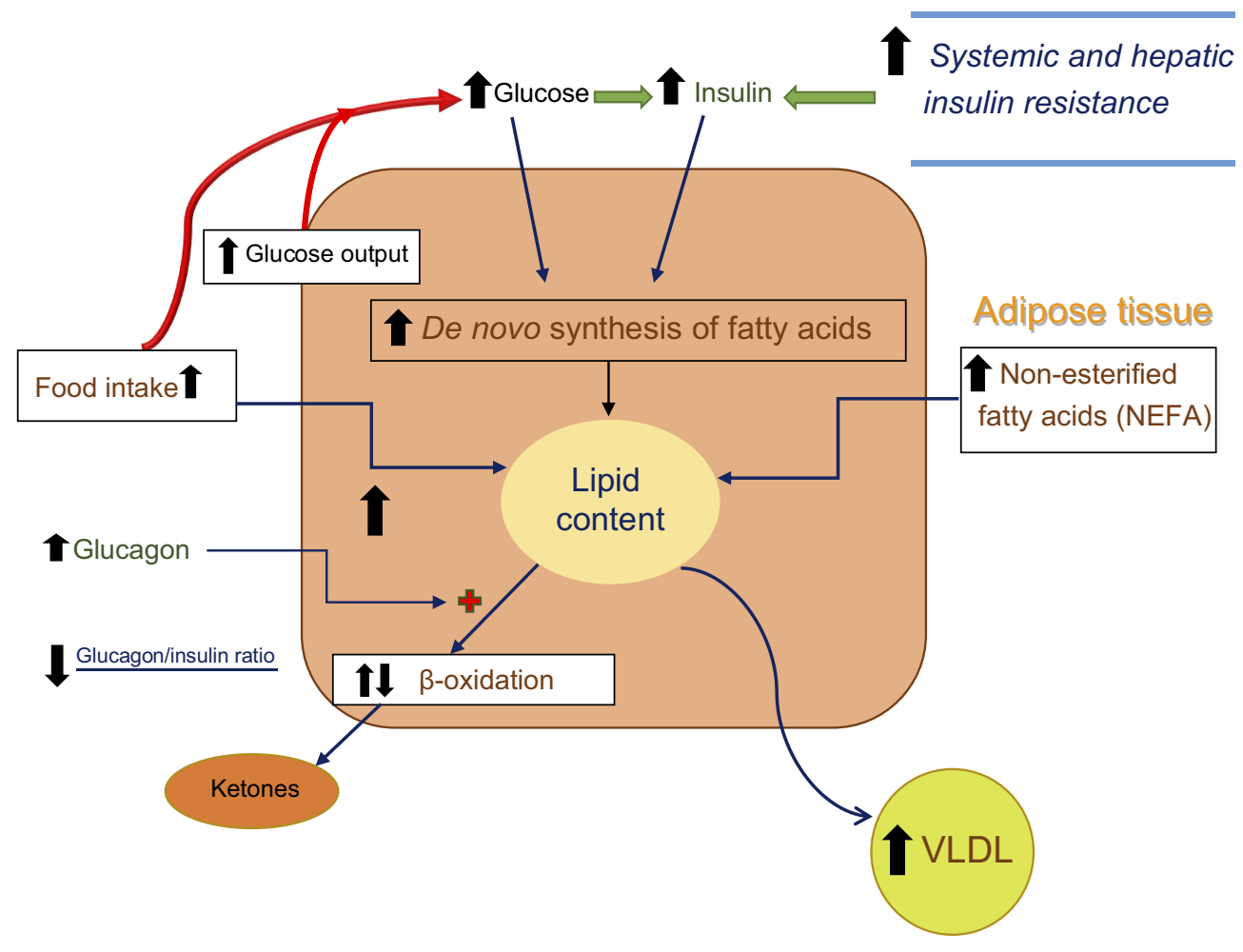

Figure 2 Lipid synthesis and deposition in hepatocytes in individuals with NAFLD irrespective of type 2 diabetes. In individuals with NAFLD (especially those with high caloric intake and obesity), hepatic glucose production is stimulated in association with increases in systemic and hepatic insulin resistance. The elevated blood glucose and the beta-cell response to the insulin resistant state both lead to increases in insulin release and blood insulin levels. As noted in Figure I, elevated blood glucose and blood insulin levels stimulate de novo fatty acid and cholesterol synthesis. Adipose tissue resistance to insulin action leads to increased release of free fatty acids from the expanded adipose tissue mass and fatty acid uptake by the liver. Glucagon levels tend to be slightly increased (but with low glucagon/insulin ratios) leading to slight or no increase in beta-oxidation. Although there is an increase in VLDL synthesis and release by the liver, the magnitude of this change appears not to be large enough to overcome the increased rate of lipid deposition.

and improve glucose control. ${ }^{16-18,48,49}$ Members of this class of agents also exhibit cardiac and renal protective qualities. ${ }^{17-19,50}$ Serious adverse events associated with their use include volume depletion and euglycemic diabetic ketoacidosis apparently related to the increased glucagon levels that result in stimulation of $\beta$-oxidation of fatty acids and production of ketones by the liver. ${ }^{36,37}$ There is also an increased risk of genital infections, urosepsis, and amputations associated with use of some members of this class of medications. ${ }^{17,18}$ Overall, the degree of weight loss with SGLT2i(s) is modest at approximately 3 kilograms; there is a net loss of body water and electrolytes during the first 4 weeks of treatment that is followed by a preferential loss of fat mass. ${ }^{20,22}$

Based on the positive effects of SGLT2i(s) on reducing body weight, total body and visceral fat mass, and reducing serum AST and ALT levels, studies were initiated to test the efficacy of these agents on NAFLD. ${ }^{15,16,28,49,51,52}$ The effect of SGLT2i(s) on the metabolic abnormalities present in patients with NAFLD (Figure 2) is depicted in Figure 3. There is some increase in food intake that is more than offset by the increased urinary calorie loss. ${ }^{53}$ The concentration of free fatty acids increases or remains unchanged. ${ }^{36,37,54}$ Treatment with SGLT2i(s) results in decreases in both glucose and insulin levels (especially in those with T2DM) which lead to a large decrease in hepatic de novo lipid synthesis. ${ }^{37}$ As noted above, glucagon-secreting alpha cells of pancreatic islets express SGLT2, and inhibition of the co-transporter (which presumably reduces intracellular glucose concentration in these cells an important signal for glucagon release) results in increased secretion and blood levels of glucagon. ${ }^{37,43,46}$ The high glucagon levels (and elevated glucagon/insulin ratio) lead to stimulation of $\beta$-oxidation (and hepatic ketone production and elevated blood ketone levels) and cause a shift from carbohydrate to fatty acid metabolism and help reduce liver triglyceride content. ${ }^{36,37,54}$ In keeping with the importance of glucagon in the pathogenesis of NAFLD, a recent study showed that while a reduction in glucagon receptor expression (using antisense technology) in patients with T2DM improved glycemic control, it also increased their ALT levels and significantly increased hepatic fat content. ${ }^{55}$ Finally, the potential effect of SGLT2i(s) on VLDL metabolism has not been studied in detail 


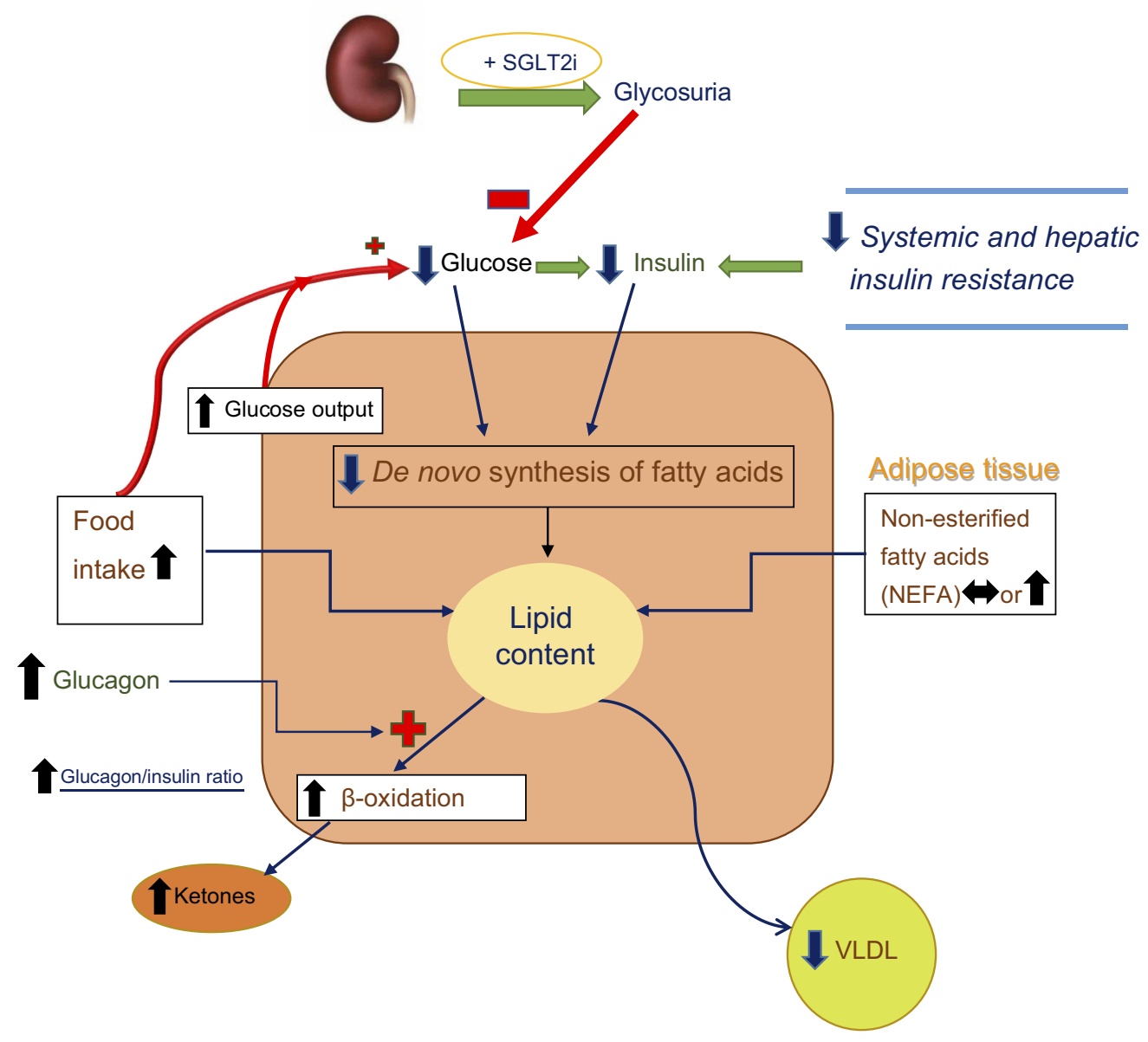

Figure 3 Lipid synthesis and deposition in hepatocytes of individuals with T2DM and NAFLD who are being treated with SGLT2 Inhibitors. Treatment of patients with T2DM and NAFLD with SGLT2i(s) lowers blood glucose (and glucotoxicity), blood insulin levels, and body mass, all leading to decreased systemic and hepatic insulin resistance. There is a reduction of de novo lipid synthesis reflecting the decreases in blood glucose and insulin levels. There is often some increase in food intake which is more than counteracted by the caloric loss due to glycosuria. There is some increase in circulating levels of non-esterified fatty acids. However, as noted in the text, SGLT2i (s) directly stimulate the release of glucagon from alpha cells of the pancreas. Elevated blood glucagon levels (and high glucagon/insulin ratios) lead to stimulation of betaoxidation (and hepatic ketone production), causing a shift from carbohydrate to fatty acid metabolism and increase blood ketones ( $\beta$-hydroxybutyrate). An increase in gluconeogenesis is noted, but the net effect on blood glucose levels is negative due to glycosuria. The sum total of the effect of SGLT2i(s) is to decrease hepatic lipid content.

in humans. In a study in rats, SGLT2i(s) were found to enhance VLDL metabolism. ${ }^{56}$ In sum, the major positive effect of SGLT2i(s) in reducing hepatic lipid content is mediated by reduction in de novo lipid synthesis (by reduced blood glucose and insulin levels) and increased beta-oxidation of fatty acids.

\section{Outcomes of studies designed to evaluate the efficacy of SGLT2i(s) on NAFLD}

Previous studies performed in human subjects to assess the safety and efficacy of SGLT2i(s) in patients with T2DM showed significant reductions in serum ALT. ${ }^{51,52}$ This effect was initially attributed to significant reductions in body mass and glycated hemoglobin $(\mathrm{HbAlc}){ }^{57}$ However, further studies showed that the reduction in liver enzymes can occur irrespective of changes in weight. ${ }^{58}$ In addition, it has been demonstrated that the reduction in body weight with the use of SGLT2i(s) is not large, and reaches a plateau rather than being sustained. ${ }^{16-18,36}$ It is therefore thought that improvements in blood liver enzyme levels are likely related to additional pathways discussed above. ${ }^{59}$

An early study examining the potential beneficial effects of the SGLT2i, Ipragliflozin, on NAFLD in patients with T2DM reported that after 4 weeks of treatment, glucose and insulin levels and the degree of insulin resistance, serum inflammatory and hepatic injury markers including Tumor Necrosis Factoralpha (TNF-alpha) and Interleukin-6 (IL-6) all decreased. ${ }^{60}$ Similar evidence was presented using Dapagliflozin in patients with T2DM and NAFLD showing improvement in inflammatory and oxidative stress markers (myeloperoxidase and reactive oxygen species). ${ }^{61}$ A retrospective study performed in patients with T2DM and NAFLD compared the effect of SGLT2i (used either Canagliflozin $100 \mathrm{mg}$ or Ipragliflozin $50 \mathrm{mg}$ ) versus 
Sitagliptin, a dipeptidyl peptidase-4 inhibitor, on body composition. $^{62}$ After 24 weeks of treatment, the groups treated with SGLT2i(s) had a reduction of body mass index compared to the group treated with Sitagliptin; this effect was independent on reductions in ALT or HbA1c. ${ }^{62}$ Evidence from other studies also suggests that effect of SGLT2i(s) on liver fat can occur irrespective of changes in glycemic control. ${ }^{15}$

Treatment with Canagliflozin reduced hepatic fat and body fat mass without significantly reducing muscle mass. ${ }^{16}$ Ipragliflozin compared with Pioglitazone showed a similar reduction in serum ALT and HbAlc; however, body weight and visceral fat were only significantly reduced in those treated with Ipragliflozin. ${ }^{63}$ Studies using Canagliflozin in patients with T2DM and NAFLD showed significant reduction in FIB-4 index values and ferritin levels (a marker of hepatic oxidative stress) suggesting improvement in hepatic fibrosis. ${ }^{64}$ In a randomized, double-blind placebo-controlled study, patients with NAFLD and T2DM were randomized to placebo, omega-3, Dapagliflozin, and a combination of both omega-3 and Dapagliflozin. Monotherapy with Dapagliflozin reduced measures of hepatocyte injury and Fibroblast Growth Factor 21 (FGF-21) consistent with a disease-modifying effect on NAFLD. ${ }^{65}$ In an interesting small study involving 24 patients with NALFD who continued to have elevated ALT levels while being treated with a GLP-1RA or Dipeptidyl Peptidase-4 inhibitors, addition of Ipragliflozin resulted in a significant reduction of ALT levels and Fibrosis Index-4 (FIB-4) in approximately half of the patients irrespective of their initial treatment; this finding suggests potential additive effect of these agents on NAFLD. ${ }^{66}$

Few studies have used liver biopsy to monitor the efficacy of SGLT2i(s) on progress or regression of NAFLD. In one study, treatment of patients with T2DM and biopsy-proven NASH with Dapagliflozin resulted in significant reductions in fasting glucose, insulin, and HbA1c levels with increased plasma adiponectin level (perhaps related to reduced visceral fat mass); this effect was seen as soon as 4 weeks of treatment. ${ }^{28}$ Results of a preliminary study using serial liver biopsies in five Japanese patients receiving Canagliflozin for 24 weeks showed remarkable histologic improvement of NASH; four patients improved from having NASH to borderline levels of disease, and one patient improved from borderline to non-NASH status. ${ }^{67}$ In addition to non-invasive measures, more studies using patients with biopsy-proven
NAFLD with or without T2DM are needed to validate the efficacy of SGLT2i(s) in treatment of NAFLD.

\section{Comparison of efficacy of SGLT2i(s) with other modes of therapy for NAFLD}

Multiple studies using a variety of agents in addition to lifestyle modifications have been tested in patients with NAFLD with and without T2DM with moderate success (Table 1). ${ }^{37,63,66-74}$ As detailed in the table, some of the studies have included one or more liver biopsies to more exactly quantify the efficacy of the employed agents and strategies. Use of Vitamin E, Pioglitazone, and GLP-1RA has shown prevention of progression and some reversal of the disease process in a subgroup of patients with NAFLD and NASH.

\section{Ongoing clinical trials assessing the effect of SGLT2i(s) on NAFLD}

It is of note that most of the evidence accrued to date on the effect of SGLT2i(s) on NAFLD has been derived from studies on rodents and from a limited number of studies in relatively small number of Japanese patients with T2DM. Table 2 summarizes ongoing and recently completed (yet unpublished) trials listed in www.ClincalTrials.gov that are focused on the potential beneficial effects of SGLT2 $i$ (s) on NAFLD. Three of the studies listed include use of liver biopsy in their protocol.

\section{Future directions and conclusions}

The accumulated evidence demonstrates the promising effect of SGLT2i(s) as a treatment modality in patients with T2DM and NAFLD. Some effects of these agents on NAFLD could be through their effect on body weight and fat mass, with weight loss being a well-established and effective strategy for control or even reversal of NAFLD. ${ }^{4,14}$ However, the overall degree of weight loss is modest and it is likely that other mechanisms detailed in Figure 3 are operative. In keeping with the possibility of additional mechanisms, it was recently shown that in obese mice with diabetes resulting from homozygous mutation of the leptin gene $\left(l e p r^{d b} / l e p r^{d b}\right)$, treatment with dapagliflozin resulted in improved glycemic control associated with improvement in arterial stiffness and alteration in their microbiome. ${ }^{76}$ Whether similar effects will be observed in patients with T2DM remains to be determined. 


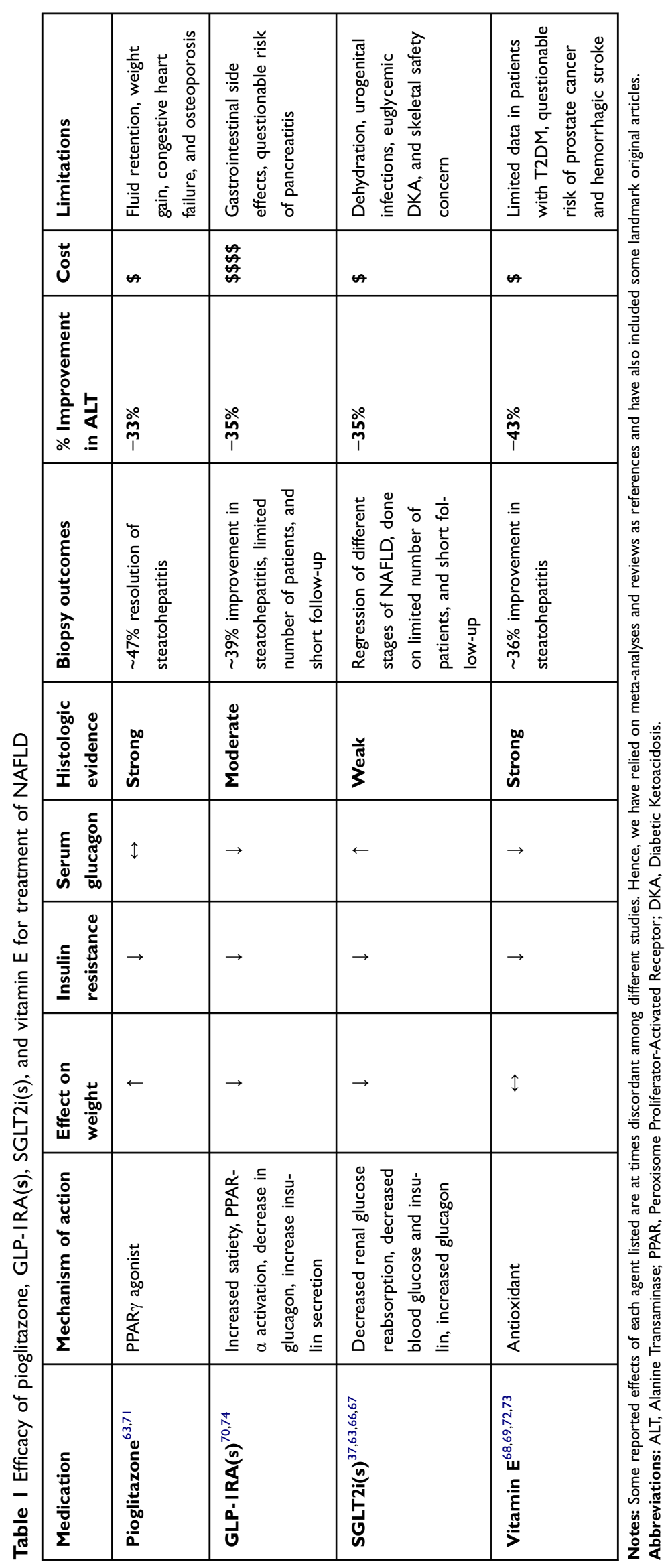




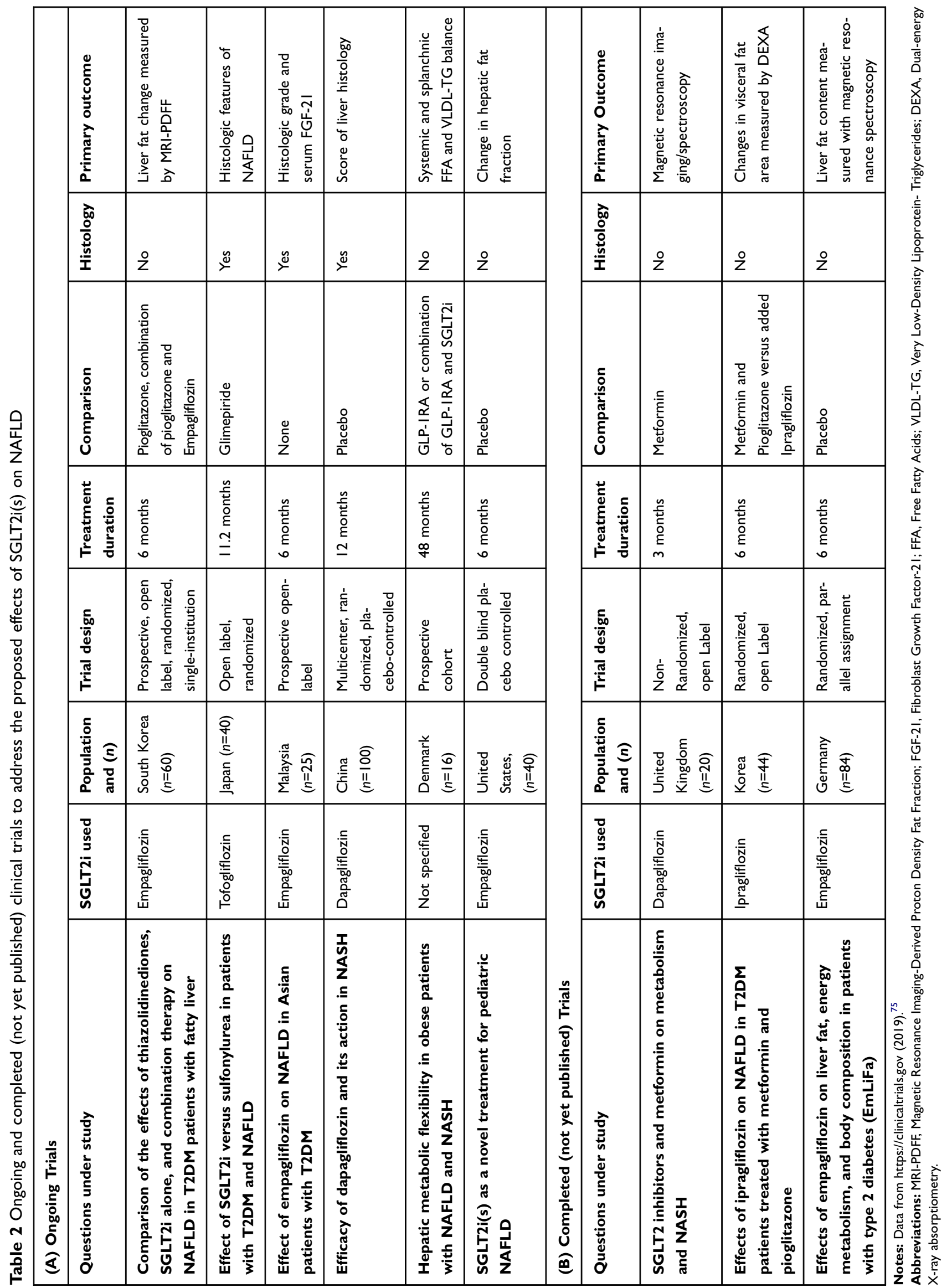


Future research could explore combination of therapeutics that have shown promising results in the treatment of NAFLD in patients with and without T2DM. Knowledge of whether the mechanisms underlying the effect of each member of the combination with other members is identical, overlapping, or additive with the other member is essential in planning such studies. One possible combination could be the use of a SGLT2 $\mathrm{i}$ added to pioglitazone. An advantage of this combination would be that the fluidretaining properties of thiazolidinediones could be nullified by the osmotic diuretic effect of the SGLT2i. Another possible combination could be a SGLT2i and a GLP-1 receptor agonist, given their different mechanisms of action (Table 1) and their probable additive effect on weight loss and glycemic control.

Finally, large-scale multicenter randomized controlled trials are necessary to examine the efficacy of SGLT2i(s) on NAFLD and NASH in patients with and without T2DM. Such studies could include use of genetic analysis and biomarkers. Further mechanistic studies would lead to better understanding of the mechanisms underlying the pathogenesis of NAFLD and the beneficial actions of SGLT2i(s).

\section{Disclosure}

The authors report no conflicts of interest in this work.

\section{References}

1. Angulo P. Nonalcoholic fatty liver disease. $N$ Engl J Med. 2002;346 (16):1221-1231. doi:10.1056/NEJMra011775

2. Michelotti GA, Machado MV, Diehl AM. NAFLD, NASH and liver cancer. Nat Rev Gastroenterol Hepatol. 2013;10(11):656-665. doi:10.1038/nrgastro.2013.183

3. Younossi ZM, Blissett D, Blissett R, et al. The economic and clinical burden of nonalcoholic fatty liver disease in the United States and Europe. Hepatology. 2016;64(5):1577-1586. doi:10.1002/hep.28785

4. Rinella ME. Nonalcoholic fatty liver disease: a systematic review. JAMA. 2015;313(22):2263-2273. doi:10.1001/jama.2015.5370

5. Van der Windt DJ, Sud V, Zhang H, Tsung A, Huang H. The effects of physical exercise on fatty liver disease. Gene Expr. 2018;18(2):89-101.

6. Vilar-Gomez E, Martinez-Perez Y, Calzadilla-Bertot L, et al. Weight loss through lifestyle modification significantly reduces features of nonalcoholic steatohepatitis. Gastroenterology. 2015;149(2):367-378.

7. Rotman Y, Sanyal AJ. Current and upcoming pharmacotherapy for non-alcoholic fatty liver disease. Gut. 2017;66(1):180-190.

8. Belfort R, Harrison SA, Brown K, et al. A placebo-controlled trial of pioglitazone in subjects with nonalcoholic steatohepatitis. $N$ Engl $J$ Med. 2006;355(22):2297-2307.

9. Sato K, Gosho M, Yamamoto T, et al. Vitamin E has a beneficial effect on nonalcoholic fatty liver disease: a meta-analysis of randomized controlled trials. Nutrition. 2015;31(7-8):923-930.

10. Abdul-Hai A, Abdallah A, Malnick SDH. Influence of gut bacteria on development and progression of non-alcoholic fatty liver disease. World J Hepatol. 2015;7(12):1679-1684. doi:10.4254/wjh.v7.i12.1679
11. Apovian CM, Aronne LJ, Bessesen DH, et al. Pharmacological management of obesity: an endocrine Society clinical practice guideline. J Clin Endocrinol Metab. 2015;100(2):342-362. doi:10.1210/jc.2014-3415

12. Zhu C, Kim K, Wang X, et al. Hepatocyte Notch activation induces liver fibrosis in nonalcoholic steatohepatitis. Sci Transl Med. 2018;10 (468):pii: eaat0344. doi:10.1126/scitranslmed.aao4496

13. Barb D, Portillo-Sanchez P, Cusi K. Pharmacological management of nonalcoholic fatty liver disease. Metabolism. 2016;65(8):1183-1195. doi:10.1016/j.metabol.2016.04.004

14. Cusi K. Treatment of patients with type 2 diabetes and non-alcoholic fatty liver disease: current approaches and future directions. Diabetologia. 2016;59(6):1112-1120. doi:10.1007/s00125-016-3952-1

15. Kuchay MS, Krishan S, Mishra SK, et al. Effect of Empagliflozin on liver fat in patients with type 2 diabetes and nonalcoholic fatty liver disease: a randomized controlled trial (E-LIFT Trial). Diabetes Care. 2018;41(8):1801-1808. doi:10.2337/dc18-0165

16. Inoue M, Hayashi A, Taguchi T, et al. Effects of canagliflozin on body composition and hepatic fat content in type 2 diabetes patients with non-alcoholic fatty liver disease. J Diabetes Investig. Epub 2018 Nov 21.

17. Zinman B, Inzucchi SE, Lachin JM, et al. Empagliflozin and cerebrovascular events in patients with type 2 diabetes mellitus at high cardiovascular risk. Stroke. 2017;48(5):1218-1225. doi:10.1161/ STROKEAHA.116.015756

18. Neal B, Perkovic V, Mahaffey KW, et al. Canagliflozin and cardiovascular and renal events in type 2 diabetes. N Engl J Med. 2017;377 (7):644-657. doi:10.1056/NEJMoa1611925

19. Ismail-Beigi F, Moghissi E, Kosiborod M, Inzucchi SE. Shifting paradigms in the medical management of type 2 diabetes: reflections on recent cardiovascular outcome trials. J Gen Intern Med. 2017;32 (9):1044-1051. doi:10.1007/s11606-017-4061-7

20. Kamei S, Iwamoto M, Kameyama M, et al. Effect of Tofogliflozin on body composition and glycemic control in Japanese subjects with type 2 diabetes mellitus. J Diabetes Res. 2018;2018:6470137. doi: $10.1155 / 2018 / 6470137$

21. Kumashiro N, Erion DM, Zhang D, et al. Cellular mechanism of insulin resistance in nonalcoholic fatty liver disease. Proc Natl Acad Sci USA. 2011;108(39):16381-16385. doi:10.1073/pnas.1113359108

22. Liu XY, Zhang N, Chen R, Zhao JG, Yu P. Efficacy and safety of sodium-glucose cotransporter 2 inhibitors in type 2 diabetes: a metaanalysis of randomized controlled trials for 1 to 2 years. J Diabetes Complications. 2015;29(8):1295-1303. doi:10.1016/j.jdiacomp.2015. 07.011

23. Shiba K, Tsuchiya K, Komiya C, et al. Canagliflozin, an SGLT2 inhibitor, attenuates the development of hepatocellular carcinoma in a mouse model of human NASH. Sci Rep. 2018;8(1):2362. doi:10.1038/s41598-018-19658-7

24. Tomic D, Kemp WW, Roberts SK. Nonalcoholic fatty liver disease: current concepts, epidemiology and management strategies. Eur $J$ Gastroenterol Hepatol. 2018;30(10):1103-1115. doi:10.1097/ MEG.0000000000001235

25. Brunt EM, Tiniakos DG. Histopathology of nonalcoholic fatty liver disease. World J Gastroenterol. 2010;16(42):5286-5296. doi:10.3748/wjg. v16.i42.5286

26. Papatheodoridi M, Cholongitas E. Diagnosis of non-alcoholic fatty liver disease (NAFLD): current concepts. Curr Pharm Des. 2018;24 (38):4574-4586. doi:10.2174/1381612825666190117102111

27. Mottin CC, Moretto M, Padoin AV, et al. The role of ultrasound in the diagnosis of hepatic steatosis in morbidly obese patients. Obes Surg. 2004;14(5):635-637. doi:10.1381/096089204323093408

28. Tobita H, Sato S, Miyake T, et al. Effects of Dapagliflozin on body composition and liver tests in patients with nonalcoholic steatohepatitis associated with type 2 diabetes mellitus: a prospective, openlabel, uncontrolled study. Curr Ther Res Clin Exp. 2017;87:13-19. doi:10.1016/j.curtheres.2017.07.002 
29. Perumpail BJ, Khan MA, Yoo ER, Cholankeril G, Kim D, Ahmed A. Clinical epidemiology and disease burden of nonalcoholic fatty liver disease. World J Gastroenterol. 2017;23(47):8263-8276. doi:10.3748/ wjg.v23.i47.8263

30. Goldberg D, Ditah IC, Saeian K, et al. Changes in the prevalence of hepatitis $\mathrm{C}$ virus infection, nonalcoholic steatohepatitis, and alcoholic liver disease among patients with cirrhosis or liver failure on the waitlist for liver transplantation. Gastroenterology. 2017;152 (5):1090-1099. doi:10.1053/j.gastro.2017.01.003

31. Baffy G, Brunt EM, Caldwell SH. Hepatocellular carcinoma in nonalcoholic fatty liver disease: an emerging menace. $J$ Hepatol. 2012;56 (6):1384-1391. doi:10.1016/j.jhep.2011.10.027

32. Larsson SC, Wolk A. Overweight, obesity and risk of liver cancer: a meta-analysis of cohort studies. Br J Cancer. 2007;97(7):1005-1008. doi:10.1038/sj.bjc.6603932

33. Day CP, James OF. Steatohepatitis: a tale of two "hits"? Gastroenterology. 1998;114(4):842-845.

34. Silverman JF, O'Brien KF, Long S, et al. Liver pathology in morbidly obese patients with and without diabetes. Am J Gastroenterol. 1990;85(10):1349-1355.

35. Horst KWT, Serlie MJ. Fructose consumption, lipogenesis, and non-alcoholic fatty liver disease. Nutrients. 2017;9(9):981. doi:10.3390/ nu9090981

36. Ferrannini E, Baldi S, Frascerra S, et al. Shift to fatty substrate utilization in response to sodium-glucose cotransporter 2 inhibition in subjects without diabetes and patients with type 2 diabetes. Diabetes. 2016;65(5):1190-1195. doi:10.2337/db15-1356

37. Daniele G, Xiong J, Solis-Herrera C, et al. Dapagliflozin enhances fat oxidation and ketone production in patients with type 2 diabetes. Diabetes Care. 2016;39(11):2036-2041. doi:10.2337/dc15-2688

38. Dongiovanni P, Stender S, Pietrelli A, et al. Causal relationship of hepatic fat with liver damage and insulin resistance in nonalcoholic fatty liver. J Intern Med. 2018;283(4):356-370. doi:10.1111/joim.12719

39. Petta S, Gastaldelli A, Rebelos E, et al. Pathology of non-alcoholic fatty liver disease. Int J Mol Sci. 2016;17(12):2082. doi:10.3390/ ijms 17122082

40. Postic C, Girard J. Contribution of de novo fatty acid synthesis to hepatic steatosis and insulin resistance: lessons from genetically engineered mice. J Clin Invest. 2008;118(3):829-838. doi:10.1172/JCI34275

41. Perry RJ, Samuel VT, Petersen KF, Shulman GI. The role of hepatic lipids in hepatic insulin resistance and type 2 diabetes. Nature. 2014;510(7503):84-91. doi:10.1038/nature13478

42. Filhoulaud G, Guilmeau S, Dentin R, Girard J, Postic C. Novel insights into ChREBP regulation and function. Trends Endocrinol Metab. 2013;24(5):257-268. doi:10.1016/j.tem.2013.01.003

43. Wang D, Luo Y, Wang X, et al. The sodium-glucose cotransporter 2 inhibitor dapagliflozin prevents renal and liver disease in western diet induced obesity mice. Int J Mol Sci. 2018;19(1):137. doi:10.3390/ ijms 19010137

44. Junker AE, Gluud L, Holst JJ, Knop FK, Vilsbøll T. Diabetic and nondiabetic patients with nonalcoholic fatty liver disease have an impaired incretin effect and fasting hyperglucagonaemia. JIM. 2016;279(5):485-493. doi:10.1111/joim.12462

45. Sala-Rabanal M, Hirayama BA, Ghezzi C, et al. Revisiting the physiological roles of SGLTs and GLUTs using positron emission tomography in mice. $J$ Physiol. 2016;594(15):4425-4438. doi:10.1113/JP271904

46. Bonner C, Kerr-Conte J, Gmyr V, et al. Inhibition of the glucose transporter SGLT2 with dapagliflozin in pancreatic alpha cells triggers glucagon secretion. Nat Med. 2015;21(5):512-517. doi:10.1038/nm.3828

47. Chen J, Williams S, Ho S, et al. Quantitative PCR tissue expression profiling of the human SGLT2 gene and related family members. Diabetes Ther. 2010;1(2):57-92. doi:10.1007/s13300-010-0006-4

48. Katsuno K, Fujimori Y, Takemura Y, et al. Sergliflozin, a novel selective inhibitor of low-affinity sodium glucose cotransporter (SGLT2). $J$ Pharmacol Exp Ther. 2007;320(1):323-330. doi:10.1124/jpet.106.110296
49. Yokonoa M, Takasua T, Hayashizakia Y, et al. SGLT2 selective inhibitor ipragliflozin reduces body fat mass by increasing fatty acid oxidation in high-fat diet-induced obese rats. Eur J Pharmacol. 2014;15(727):66-74.

50. Wiviott SD, Raz I, Bonaca MP, et al. Dapagliflozin and cardiovascular outcomes in type 2 diabetes. $N$ Engl J Med. 2019;380(4):347-357.

51. Lavalle-González FJ, Januszewicz A, Davidson J, et al. Efficacy and safety of canagliflozin compared with placebo and sitagliptin in patients with type 2 diabetes on background metformin monotherapy: a randomised trial. Diabetologia. 2013;56(12):2582-2592.

52. Bailey CJ, Gross JL, Pieters A, Bastien A, List JF. Effect of dapagliflozin in patients with type 2 diabetes who have inadequate glycaemic control with metformin: a randomised, double-blind, placebo-controlled trial. Lancet. 2010;375(9733):2223-2233.

53. Komiya C, Tsuchiya K, Shiba K, et al. Ipragliflozin improves hepatic steatosis in obese mice and liver dysfunction in type 2 diabetic patients irrespective of body weight reduction. PLoS One. 2016;11(3):1-19.

54. Yokono M, Takasu T, Hayashizaki Y, et al. SGLT2 selective inhibitor ipragliflozin reduces body fat mass by increasing fatty acid oxidation in high-fat diet-induced obese rats. Eur J Pharmacol. 2014;727:66-74.

55. Morgan ES, Tai L-J, Pham NC, et al. Antisense inhibition of glucagon receptor by IONIS-GCGR rx improves type 2 diabetes without increase in hepatic glycogen content in patients with type 2 diabetes on stable metformin therapy. Diabetes Care. 2019;42:4.

56. Basu D, Huggins LA, Scerbo D, et al. Mechanism of increased LDL (Low-density lipoprotein) and decreased triglycerides with SGLT2 (Sodium-Glucose Cotransporter 2) inhibition. ATVB. 2018;38 (9):2207-2216

57. Leiter LA, Forst T, Polidori D, Balis DA, Xie J, Sha S. Effect of canagliflozin on liver function tests in patients with type 2 diabetes. Diabetes Metab. 2016;42(1):25-32.

58. Sattar N, Fitchetts D, Hantel S, Jyothis T, Zinman G, Zinnman B. Empagliflozin is associated with improvements in liver enzymes potentially consistent with reductions in liver fat: results from randomised trials including the EMPA-REG OUTCOME® trial. Diabetologia. 2018;61(10):2155-2163.

59. Lee PCH, Gu Y, Yeung MY, et al. Dapagliflozin and empagliflozin ameliorate hepatic dysfunction among chinese subjects with diabetes in part through glycemic improvement: a single-center, retrospective, observational study. Diabetes Ther. 2018;9(1):285-295.

60. Tahara A, Kurosaki E, Yokono M, et al. Effects of SGLT2 selective inhibitor ipragliflozin on hyperglycemia, hyperlipidemia, hepatic steatosis, oxidative stress, inflammation, and obesity in type 2 diabetic mice. Eur J Pharmacol. 2013;715(1-3):246-255.

61. Tang L, Wu Y, Tian M, et al. Dapagliflozin slows the progression of the renal and liver fibrosis associated with type 2 diabetes. $\mathrm{Am} \mathrm{J}$ Physiol Endocrinol Metab. 2017;313:563-576.

62. Seko Y, Sumida Y, Tanaka S, et al. Effect of sodium glucose cotransporter 2 inhibitor on liver function tests in Japanese patients with non-alcoholic fatty liver disease and type 2 diabetes mellitus. Hepatol Res. 2016;47:1072-1078.

63. Ito D, Shimizu S, Inoue K, et al. Comparison of ipragliflozin and pioglitazone effects on nonalcoholic fatty liver disease in patients with type 2 diabetes: a randomized, 24-week, open-label, activecontrolled trial. Diabetes Care. 2017;40(10):1364-1372.

64. Itani T, Ishihara T. Efficacy of canagliflozin against nonalcoholic fatty liver disease: a prospective cohort study. Obes Sci Pract. 2018;4(5):477-482.

65. Eriksson JW, Lundkvist P, Jansson PA, et al. Effects of dapagliflozin and $n-3$ carboxylic acids on non-alcoholic fatty liver disease in people with type 2 diabetes: a double-blind randomised placebocontrolled study. Diabetologia. 2018;61(9):1923-1934.

66. Ohki T, Isogawa A, Toda N, Tagawa K. Effectiveness of ipragliflozin, a sodium-glucose co-transporter 2 inhibitor, as a second-line treatment for non-alcoholic fatty liver disease patients with type 2 diabetes mellitus who do not respond to incretin-based therapies including glucagon-like peptide. Clin Drug Investig. 2016;36(4):313-319. 
67. Akuta N, Watanabe C, Kawamura Y, et al. Effects of a sodiumglucose cotransporter 2 inhibitor in nonalcoholic fatty liver disease complicated by diabetes mellitus: preliminary prospective study based on serial liver biopsies. Hepatol Commun. 2017;1:46-52.

68. Schürks M, Glynn RJ, Rist PM, Tzourio C, Kurth T. Effects of vitamin $\mathrm{E}$ on stroke subtypes: meta-analysis of randomised controlled trials. BMJ. 2010;341:c5702.

69. Sanyal AJ, Chalasani N, Kowdley KV, et al. Pioglitazone, vitamin E, or placebo for nonalcoholic steatohepatitis. $N$ Engl J Med. 2010;362:1675-1685.

70. Armstrong MJ, Gaunt P, Aithal GP, et al. Liraglutide safety and efficacy in patients with non-alcoholic steatohepatitis (LEAN): a multicentre, double-blind, randomised, placebo-controlled phase 2 study. Lancet. 2016;387:679-690.

71. Aithal GP, Thomas JA, Kaye PV, et al. Randomized, placebo-controlled trial of pioglitazone in nondiabetic subjects with nonalcoholic steatohepatitis. Gastroenterology. 2008;135(4):1176-1184.
72. Bhathena SJ, Berlin E, Judd JT, et al. Effects of $\omega 3$ fatty acids and vitamin $\mathrm{E}$ on hormones involved in carbohydrate and lipid metabolism in men. Am J Clin Nutr. 1991;54(4):684-688.

73. Klein EA, Thomson IM Jr, Tangen CM, et al. Vitamin E and the risk of prostate cancer: updated results of the selenium and vitamin $\mathrm{E}$ cancer prevention trial. JAMA. 2011;306(14):1549-1556.

74. Marso SP, Daniels GH, Brown-Frandsen K, et al. Liraglutide and cardiovascular outcomes in type 2 diabetes. $N$ Engl J Med. 2016;375 (4):311-322.

75. U.S. National Library of Medicine [homepage on the Internet]. Available from: https://clinicaltrials.gov/. Accessed June 27, 2019.

76. Lee DM, Battson ML, Jarrell DK, et al. SGLT2 inhibition via dapagliflozin improves generalized vascular function and alters the gut microbiota in type 2 diabetic mice. Cardiovasc Diabetol. 2018;17:62.

\section{Publish your work in this journal}

Diabetes, Metabolic Syndrome and Obesity: Targets and Therapy is an international, peer-reviewed open-access journal committed to the rapid publication of the latest laboratory and clinical findings in the fields of diabetes, metabolic syndrome and obesity research. Original research, review, case reports, hypothesis formation, expert opinion and commentaries are all considered for publication. The manuscript management system is completely online and includes a very quick and fair peer-review system, which is all easy to use. Visit http://www.dovepress.com/testimonials.php to read real quotes from published authors. 